\title{
Chronisch-entzündliche Erkrankungen
}

\section{Interdisziplinäre Versorgung im Fokus}

Für die Therapie chronisch entzündlicher Erkrankungen stehen verschiedene Medikamente zur Verfügung, darunter auch Biologika. Neben einer geeigneten Behandlung kommt dem interdisziplinären Versorgungsansatz eine besondere Bedeutung zu. Um diesen zu fördern, wurden „Inflammation Center“ gegründet, in denen eine interdisziplinäre Zusammenarbeit stattfindet.

Chronisch entzündliche Erkrankungen werden heute als Systemerkrankungen verstanden. Sie können sich in verschiedenen Organsystemen manifestieren. Immuntherapien bieten hier einen vielversprechenden Ansatz: Beispielsweise wurde das Biologikum Ustekinumab (Stelara ${ }^{\circledR}$ ), das die Interleukine-12/23 hemmt, bereits für vier Indikationen zugelassen: Plaque-Psoriasis, Psoriasis-Arthritis, Morbus Crohn und Colitis ulcerosa. Und der selektive Interleukin-23-Hemmer Guselkumab (Tremfya ${ }^{\circledR}$ ) ist für die Plaque-Psoriasis und Psoriasis-Arthritis zugelassen.

\section{Deutsche Inflammation Center} Initiative fördert interdisziplinären Austausch

Zusätzlich zu einer geeigneten Therapie benötigen Patienten einen interdisziplinären Versorgungsansatz. Um die interdisziplinäre Entzündungsme- dizin weiter voranzubringen, wurde in Deutschland die Inflammation Center Initiative (ICl) gegründet. Der Initiative gehören bereits Ärzte aus der Gastroenterologie, der Dermatologie und der Rheumatologie aus zwölf universitären Entzündungszentren an, berichtete Prof. Dr. Knut Schäkel, Stellvertretender Klinikdirektor der Hautklinik, Universitätsklinikum Heidelberg. In den beteiligten Inflammation Centern arbeiten die verschiedenen Fachdisziplinen bereits bei komplexen chronisch-entzündlichen Erkrankungen zusammen. Allerdings unterscheiden sich die bestehenden formalen Strukturen sowie die Häufigkeit von interdisziplinären Sprechstunden oder Entzündungsboards zum Teil noch stark.

Bisherige Projekte der ICI umfassen die Erhebung des Status quo mittels leitfadengestützter Interviews, die Erarbeitung einer Diskussionsgrundlage für die optimalen Strukturen, Prozesse und Abläufe in Entzündungszentren und einer idealen Versorgungsstruktur sowie die Analyse von Krankenkassendaten zum Status quo der Regelversorgung und der Struktur der Entzündungszentren. Die Veröffentlichungen dazu werden vorbereitet, so der Referent.

Bericht:Dr. Miriam Sonnet

Hinweis des Verlags. Der Verlag bleibt in Hinblick auf geografische Zuordnungen und Gebietsbezeichnungen in veröffentlichten Karten und Institutsadressen neutral.

rheuma plus $2021 \cdot 20: 259$

https://doi.org/10.1007/s12688-021-

00476-1

(c) Springer-Verlag GmbH Austria, ein Teil von Springer Nature 2021
Quelle: Digitales

Pressegespräch der Janssen-Cilag GmbH Deutschland zum Inflammation Summit "Versorgung von Patient:innen mit chronisch-entzündlichen Erkrankungen - Janssen's Engagement in der Immunologie"; 28.09.2021

Hier steht eine Anzeige. 